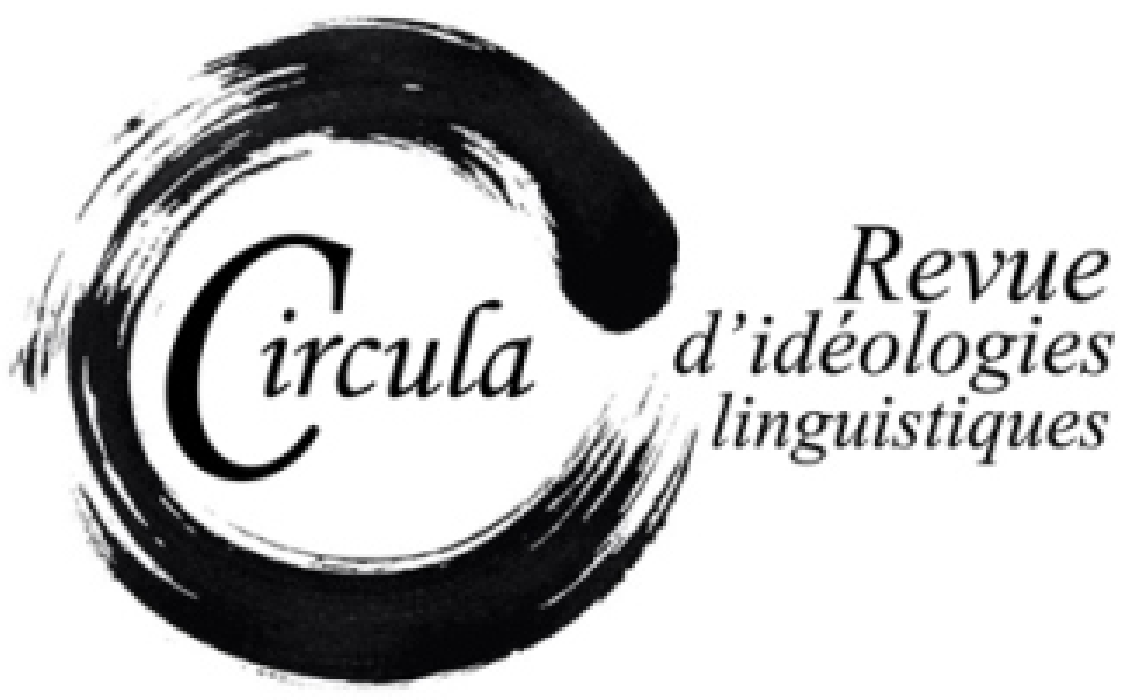

TITRE: ITALIANO DELLA RETE NELLA STAMPA ITALIANA

Auteur(s): GiUliana FIORENTINo, UniVERSITÀ del Molise

ReVue: CIRCULA, NUMÉRO 2, PAGES 176-191

ISSN: 2292-8235

Directeurs: Wim Remysen et SABINE SCHWARZE

URI: HTTP://HDL.HANDLE.NET/11143/7982

DOI: $11143 / 7982$ 


\title{
Italiano della rete nella stampa italiana
}

\author{
Giuliana Fiorentino (Università del Molise) \\ giuliana.fiorentino@unimol.it
}

\begin{abstract}
Riassunto: Il contributo si propone di analizzare il modo in cui la stampa italiana presenta e discute aspetti evolutivi del cosiddetto italiano dei nuovi media. Lo studio prende le mosse da una serie di ricerche ed osservazioni condotte sull'inglese americano e sulla cultura linguistica delle nuove generazioni da Crispin Thurlow (2003, 2005, 2009). Lo stesso Thurlow (2006) realizza un'analisi della posizione della stampa americana rispetto alla lingua di internet e alla sua influenza sulla capacità espressiva degli adolescenti americani. La ricerca illustra come molti articoli usciti sulla stampa italiana negli ultimi anni abbiano assunto una posizione quasi sempre preoccupata e denigratoria rispetto ai nuovi media e ai nativi digitali. In particolare la stampa italiana considera negativamente l'effetto che la diffusione dei nuovi media della comunicazione avrebbe sulla competenza linguistica soprattutto delle nuove generazioni. Il contributo prende altresì in esame i tratti linguistici dell'italiano emergente nei nuovi media che vengono maggiormente descritti e sanzionati dalla stampa (la diffusione delle abbreviazioni e degli emoticon, l'utilizzo di grafie non standard, ecc.) e che contribuiscono a diffondere una certa immagine della lingua di internet trascurando altri aspetti invece positivi e che rivelano una creatività notevole.
\end{abstract}

Parole chiave: ideologia linguistica; e-italiano; lingua standard e non standard; livello grafico; ortografia

Abstract: The paper analyzes how the Italian press presents and discusses the evolutionary aspects of the language of the so-called new media. The study builds on a series of research and observations conducted on American culture and the language of American new generations by Crispin Thurlow (2003, 2005, 2009). The same Thurlow (2006) realizes an analysis of the position of the American press on the language of the Internet and its influence on the communication attitudes of American teenagers. Our research shows how many articles published in the Italian press in recent years have taken a position almost always worried regarding the new media and the digital natives. In particular, the Italian press considers negatively the effect of new media on the linguistic competence especially of the younger generation. The contribution will also take into consideration the linguistic features emerging in the new media and will notice which of them are better described and frequently sanctioned by the press (the spread of abbreviations and emoticons, the use of non-standard spellings, etc.).

Keywords: linguistic ideology; e-Italian; standard and non standard language; graphic level; orthography 


\section{Introduzione}

La stampa italiana presenta e discute gli usi linguistici diffusi nei nuovi media (media digitali) in una luce spesso negativa. La lingua utilizzata nella rete viene inoltre accomunata alla lingua degli SMS, con tutte le caratteristiche che in quest'ultima vengono spesso evidenziate e sanzionate ${ }^{1}$. In questo lavoro mi propongo due obiettivi connessi ma distinti: il primo è analizzare il tipo di ideologia che i media a stampa diffondono sulla lingua italiana usata in rete e il secondo è individuare quale sia il tipo di lingua che i giornali considerano «l'italiano della rete».

In particolare dimostrerò come siano vere anche per la situazione italiana due ipotesi già osservate da altri studi in merito all'inglese (Thurlow, 2005, 2006, 2009), e cioè che a) l'ideologia linguistica che la stampa costruisce rispetto ai media digitali scaturisce da un presunto «determinismo tecnologico» e che b) i (meta)discorsi della stampa contribuiscono a loro volta al processo di «registrazione» (traduco in questo modo il termine enregisterment usato nella sociolinguistica statunitense, cf. Squires, 2010) di una nuova varietà di lingua, l'italiano della rete, appunto.

Queste due ipotesi scaturiscono da un lato dalla semplice esperienza di lettrice di giornali sensibile al tema dell'evoluzione della lingua - soprattutto in chiave pedagogica - e dall'altro lato da specifici interessi di ricerca che da anni mi portano a descrivere e studiare le possibili peculiarità linguistiche legate alla variabilità diamesica e al particolare formato di produzione della comunicazione in rete.

Chiarirò fra un attimo che cosa intendo per determinismo tecnologico e per registrazione (cf. paragrafo 4) di una nuova varietà di lingua. Prima di addentrarmi nello specifico del mio ragionamento, spiegherò brevemente che cosa intendo per ideologia linguistica e che strumenti teorici adotterò nel corso di questo lavoro (cf. paragrafo 2).

\footnotetext{
1. La ricerca linguistica si è occupata ampiamente negli scorsi decenni della scrittura di sms. L'inglese, per definire la scrittura di messaggi di testo col cellulare o la comunicazione via sms, ha coniato il termine texting, che il Merriam Webster online definisce come verbo transitivo col significato di «to send a text message from one cell phone to another», e come verbo intransitivo col significato di «to communicate by text messaging». La pratica di scrittura del texting è stata tipicamente associata all'uso di abbreviazioni e di emoticon, ma le ricerche in questa direzione si esauriranno probabilmente molto presto. Infatti la diffusione dei telefoni cosiddetti smartphone a tecnologia avanzata, con i quali ci si connette alla rete internet, ha fatto calare drasticamente l'uso degli sms a vantaggio di altre modalità di invio di testi brevi di messaggistica mediante telefono. Questi sistemi che sfruttano la connessione ad internet non hanno limiti di caratteri, non comportano costi aggiuntivi e vengono scritti con varie applicazioni (la più famosa e utilizzata attualmente è whatsapp) avendo a disposizione ampi vocabolari e sistemi di scrittura facilitata che anticipano la forma delle parole (talvolta anche correggendola) per cui il ricorso ad abbreviazioni è drasticamente calato.
} 


\section{Ideologia linguistica: definizioni}

Le definizioni di ideologia linguistica a cui farò riferimento verranno considerate con particolare riferimento alla lingua dei giovani e alle culture giovanili (per una visione critica della cultura linguistica delle nuove generazioni i riferimenti sono i già citati lavori di Thurlow, 2003, 2005, 2009; cf. qui paragrafo 3).

La parola ideologia ha una storia complessa ma è possibile schematicamente individuare almeno due accezioni (Blommaert, 2006, alla voce «Language ideology» in Encyclopedia of Language \& Linguistics). La prima è quella che si consolida nell'ambito della tradizione marxista. L'ideologia viene definita come un insieme di idee e interessi di un particolare gruppo sociale e si connota negativamente perché collegata a concetti di dominio e potere.

La seconda accezione è quella delle scienze sociali, da Durkheim (1895) in poi, e annovera l'ideologia nell'ambito dei sistemi di credenze e visioni del mondo. In questo secondo senso non c'è un'accezione negativa, bensì una connotazione neutra e l'ideologia viene definita come l'insieme di assunzioni non confessate che accomuna un gruppo di persone (Blommaert, 2006).

In questo articolo il concetto di ideologia verrà utilizzato nell'accezione delle scienze sociali. Ma anche in questo secondo caso occorre precisare che il termine talvolta ha assunto un'accezione peggiorativa in quanto all'ideologia intesa come insieme di credenze si è associata l'idea che tali credenze siano basate non su fatti, bensì spesso su pregiudizi. Da questo deriva che l'ideologia sia percepita come fallace e venga contrapposta alle teorie scientifiche, che sono invece considerate oggettive perché basate su dati. Nel seguito del discorso utilizzerò il concetto di ideologia in un'accezione il più possibile neutra.

Una storia ulteriore è quella del concetto di ideologia linguistica o language ideology, nozione che nasce nell'ambito dell'antropologia linguistica (Blommaert, 2006), ma che a sua volta non è scevra da accezioni peggiorative o comunque definizioni che negano la possibilità che un'ideologia possa essere neutra².

Per quanto concerne la nozione di ideologia linguistica - utilizzata in particolare nell'ambito dell'antropologia linguistica - essa viene spesso definita e discussa a partire dai lavori di Silverstein $(1977,1979,1992)$ e poi di Woolard $(1992,1998)$ ed infine di Blommaert $(2006)$.

Nel lavoro del 1979 Silverstein discute soprattutto il rapporto tra ideologie linguistiche e strutture del linguaggio, con un'analisi dettagliata di tre esempi in cui i due aspetti sono interconnessi e il

2. Gal (osserva come la parola ideologia in riferimento alle idee che i parlanti hanno sulla lingua e sulle differenze linguistiche sia intenzionalmente polemica «in order to highlight the fact that these ideas are always positioned in some way, relate to politics, and are influenced by power. There is no "view from nowhere" no opinions about language that are not in some way "neutral or only scientific"» (Gal, 2002: 198). 
cambiamento linguistico viene spiegato alla luce di fattori ideologici che vengono in un certo senso grammaticalizzati in precise strutture linguistiche. L'articolo del 1998 invece traccia e definisce in modo ancora più utile e ampio la storia del termine ideologia.

Il convegno annuale del 1991 dell'associazione americana di antropologia era dedicato proprio alla definizione del concetto di ideologia linguistica. Kathryn Woolard (1992) individua quattro concetti chiave che definiscono questo termine e che si ritrovano in quasi tutte le definizioni utilizzate in letteratura.

I punti condivisi tra le varie definizioni di ideologia linguistica sono: il fatto che l'ideologia è sempre concepita come un insieme ideazionale, il fatto che è legata a posizioni sociali specifiche e non è ritenuta neutra, il fatto che costituisce una visione distorta delle cose, il fatto che è legata alla legittimazione del potere sociale (cf. Woolard, 1992: 237-238). Mentre in questo lavoro del 1992 Woolard sembra fare propria l'idea che l'ideologia sia un sistema coerente ed esplicito, espresso cioè in modo conscio da una comunità, successivamente sembra cambiare questa posizione, sulla quale, del resto, non c'è uniformità di giudizio neppure oggi.

In uno studio successivo, che cerca di sistematizzare l'ideologia linguistica in quanto campo di studi autonomo, Woolard (1998) torna di nuovo sul confronto di varie definizioni di ideologia linguistica. Ripropongo qui di seguito le cinque definizioni:

1. «Sets of beliefs about language articulated by users as a rationalization or justification of perceived language structure and use» [«Insieme di credenze sul linguaggio articolate dai parlanti come razionalizzazione o giustificazione della struttura della lingua e dell'uso percepiti»] (Silverstein, 1979: 193);

2. «Self-evident ideas and objectives a group holds concerning roles of language in the social experiences of members as they contribute to the expression of the group» [«ldee e obbiettivi manifesti che un gruppo coltiva a proposito dei ruoli della lingua nelle esperienze sociali dei membri allorché contribuiscono all'espressione del gruppo»] (Heath, 1989: 53);

3. «Cultural system of ideas about the social and linguistic relationship, together with their loading of moral and political interests» [«Sistema culturale di idee sulla relazione sociale e linguistica, insieme con il loro carico d'interessi morali e politici»] (Irvine, 1989: 255);

4. «Shared bodies of commonsense notions about the nature of language in the world» [«lnsiemi condivisi di nozioni di senso comune sulla natura della lingua nel mondo»] (Rumsey, 1990: 346);

5. «Representations, whether explicit or implicit, that construe the intersection of language and human beings in a social world» [Rappresentazioni, siano esse esplicite o implicite, che costruiscono l'intersezione di lingua e degli esseri umani in un mondo sociale»] (Woolard, 1998: 3) 
Intrecciando e confrontando le cinque definizioni presentate da Woolard emerge una definizione, che adotterò nel corso di questa ricerca, secondo cui l'ideologia linguistica consiste nelle idee che un gruppo sociale o un'intera società avanzano rispetto a una lingua o a una sottovarietà di una lingua.

L'aspetto più interessante della definizione adottata è che l'ideologia linguistica non necessariamente analizza o rispecchia caratteristiche oggettive della lingua o della varietà in esame, ma piuttosto rispecchia ciò che un gruppo di individui pensa (idee) della lingua e di tali caratteristiche. Inoltre questa definizione punta decisamente sulla rappresentazione collettiva (in vario modo intesa) di una lingua o varietà di lingua e non su ciò che i singoli individui pensano.

Le cinque definizioni presentano anche delle interessanti differenze: alcuni autori ritengono che la costruzione dell'ideologia linguistica passi attraverso le dichiarazioni esplicite e ufficiali di una data collettività (sostanzialmente questa è la posizione di Silverstein), mentre altri autori ritengono che anche gli atteggiamenti impliciti rispetto alla lingua siano da considerare parte integrante della costruzione di un'ideologia linguistica. Questa seconda posizione si è fatta sempre più spazio tra i ricercatori. L'inclusione dell'elemento inconscio o implicito come rappresentazione che costruisce l'ideologia linguistica ha delle importanti ricadute rispetto alla metodologia di analisi. Nel caso in cui la costruzione dell'ideologia linguistica si intenda inclusiva anche delle rappresentazioni implicite, infatti, l'analista dovrà considerare dati più eterogenei e si riserverà un margine di interpretazione dei dati più ampio. La raccolta e valutazione di questo tipo di dati non espliciti non è di per sé facile (cf. su questo tema la discussione in Leone, 2011).

Ai fini di questa ricerca ho ritenuto di lavorare sulla ideologia linguistica della stampa utilizzando solo rappresentazioni esplicite, cioè articoli e commenti diretti sul tema dei media digitali e del loro impatto sulla cultura e sulla lingua delle nuove generazioni.

\section{Giovani e adolescenti come costruzione degli adulti}

La ricerca si ispira al lavoro svolto da Thurlow (2006), il quale analizza la posizione della stampa americana rispetto all'inglese della rete e all'influenza della rete sulla capacità espressiva degli adolescenti (13-19) anglofoni3.

Secondo Thurlow la stampa americana rappresenta la lingua della comunicazione mediata dal computer come una varietà di lingua stravagante e caricaturale rispetto alle pratiche reali. Ne vengono esagerate ed esasperate le differenze rispetto ad altre varietà del discorso. I metadiscorsi dei giornali inoltre non riescono a comprendere la linea evolutiva del cambiamento linguistico. Thurlow

3. Il corpus di circa 100 articoli analizzato da Thurlow è stato assemblato come segue: «With the help of two research assistants, the ProQuest and LexisNexis, newspaper databases were searched for any English-language news articles between 2001 and 2005 covering issues related to young people, language, and new technology. (Search terms included: language, teenagers, adolescents, adolescence, youth, young people, technology, email, text messaging, instant messaging)» (Thurlow, 2006: 671). L'analisi è condotta in modo qualitativo seguendo l'approccio della Critical Discourse Analysis. 
rileva infine come l'ideologia linguistica relativa alle nuove tecnologie sia uno dei tanti aspetti sui quali i media americani costruiscono la loro ideologia dell'adolescenza e dell'essere giovani. Se ci si vuole davvero occupare degli adolescenti e dei giovani occorre sgombrare il campo dalle tante inesattezze e sfidare gli stereotipi sugli adolescenti e sulle loro pratiche comunicative (Thurlow, 2005: 7).

Sulla falsariga delle indagini di Thurlow anche all'interno della nostra ricerca si è ritenuto utile tener conto del modo in cui i media costruiscono il concetto di adolescenti e giovani. Come si vedrà in sede di analisi dei dati italiani, l'ideologia linguistica che definisce l'impatto dei nuovi media sulle capacità espressive e sulla diffusione di una «presunta» lingua della rete si intreccia anche in Italia con il tema più ampio della rappresentazione del mondo giovanile e adolescenziale e con l'ideologia linguistica che attribuisce a giovani e adolescenti una differente modalità comunicativa, quasi sempre inferiore e manchevole rispetto alla modalità comunicativa degli adulti.

Ma chi sono gli adolescenti? L'adolescenza, a ben considerare, è un costrutto economico e istituzionale, è ciò che gli adulti dicono che sia (Thurlow, 2005: 1). Benché non esista un concetto monolitico di cultura adolescenziale e giovanile, spesso gli stereotipi su giovani e adolescenza si basano proprio su una «presunta» monodimensionalità dell'essere teenager (dove per teenager si intende la fascia di età compresa tra i 13 e i 19 anni). L'adolescenza nei media americani è dipinta in modi irrealisticamente uniformi, largamente negativi e infine unidimensionali (Thurlow, 2005: 8). Sembrerebbe, sempre secondo Thurlow, che la società abbia messo in atto un vero e proprio attacco alla gioventù (Thurlow, 2005: 10).

Nell'ambito di questo «attacco alla gioventù» un posto di particolare rilievo è occupato dal tema della comunicazione, cioè di come comunicano i giovani. La comunicazione di giovani e adolescenti assume grande importanza negli studi sociali per il fatto che in queste fasce di età, soprattutto tra i teenager, la relazione tra pari occupa un ruolo centrale e la comunicazione è lo strumento principale della costruzione delle relazioni.

Thurlow osserva come la stampa descrivendo le caratteristiche della comunicazione di giovani e adolescenti tipicamente le collega alla diffusione delle tecnologie digitali. Le opinioni più comuni su giovani e comunicazione sono cosi sintetizzate:

- la mancanza di volontà di comunicare da parte dei giovani;

- l'incomprensibilità da parte degli adulti di quanto comunicano i giovani;

- le forti limitazioni della capacità comunicativa nei giovani;

- l'ignoranza e la non consapevolezza da parte dei giovani delle pratiche della (buona) comunicazione (Thurlow, 2005: 9). 
Per contrastare l'attacco ai giovani (youth attack) e per diffondere una visione più corretta della comunicazione di giovani e adolescenti Thurlow propone di andare oltre gli stereotipi e considerare le pratiche creative degli adolescenti realizzate mediante i nuovi media. A questo tema è dedicato un numero monografico del Journal of Computer-Mediated Communication che presenta nove studi su usi creativi del web e pratiche culturali messe in atto dai giovani (per un'introduzione al volume si veda Thurlow, 2009). In un altro studio infine Thurlow presenta la comunicazione dei giovani dal di dentro, secondo la loro prospettiva (Thurlow, 2003).

Nello sforzo di combattere gli stereotipi legati al mondo giovanile e al web Thurlow sottopone a vaglio critico anche la validità della nozione di net generation (Thurlow e McKay, 2003). Anche in questo ambito i media dipingono le nuove generazioni come internet dipendenti e peccano - ancora una volta - di un atteggiamento fortemente semplicistico (Thurlow e McKay, 2003: 95). Per contrastare questo ennesimo pregiudizio Thurlow propone di studiare in modo più approfondito e ampio la comunicazione giovanile e di farlo anche partendo dalla prospettiva dei giovani - quindi in un approccio per cosi dire etnografico - e non solo partendo dal punto di vista degli adulti.

\section{Registrazione della varietà definita «lingua della rete»}

Per collocare opportunamente il tema dell'ideologia linguistica relativamente alla varietà di lingua definita «lingua della rete» (o con etichette simili), occorre capire che cosa definiamo «lingua della rete».

La nozione di lingua delle rete è di per sé problematica e si fa fatica a individuare una varietà di lingua i cui tratti linguistici siano specifici ed esclusivi della comunicazione mediata dal computer (d'ora in poi CMC) in quanto tale. Non è questa la sede per una discussione tanto ampia, per cui mi limito a rimandare all'analisi di questo argomento che ho esposto in Fiorentino (2013) dove peraltro concludo che:

[...] the Internet language emerges not properly as the manifestation of a set of well identifiable linguistic features. Nonetheless we claim that typographic and orthographic features that have been listed in the previous paragraphs strongly characterise and identify the language on the web as a «register». [...] For the Internet language the peculiar features are typographic features (like emoticons, abbreviations, acronyms, expressive punctuation, simplification of punctuation conventions) which have been over-generalised in informal and interactive texts (chat, IM, blog, social networks). Other features like syntactic and lexical choices are not peculiar of this register and overlap with other linguistic varieties like informal speech or italiano popolare [...]. (Fiorentino, 2013: 89) 
Nonostante la difficoltà dei linguisti a definire in modo univoco la lingua della rete e se ne esista una, è interessante valutare come l'immaginario collettivo percepisca gli usi linguistici del web e in generale la CMC e come tenda a identificare una varietà di lingua unitaria. A questo proposito si osserva come i tratti linguistici dell'italiano della CMC facilmente individuati e maggiormente sanzionati dalla stampa vengano poi assunti in modo stereotipato. La stampa contribuisce cioè a diffondere una immagine - negativa - della lingua della rete e spesso trascura altri aspetti che invece rivelano una certa creatività degli usi linguistici che il web rende possibili. I tratti più spesso menzionati e criticati - come già anticipato nella citazione - sono le abbreviazioni, le emoticon, l'utilizzo di grafie non standard.

In altre parole, la stampa e i media in genere (insieme al sistema educativo del paese) stanno contribuendo alla registrazione della lingua dei media digitali e lo stanno facendo secondo un certo cliché. Vediamo in dettaglio che cosa sta accadendo e che cosa significa «registrazione» di una varietà di lingua.

L'idea che internet influenzi la lingua è un luogo comune nei discorsi pubblici (Squires, 2010: 457). In molti paesi circolano termini come netspeak, netlingo o chatspeak, cioè parole che individuerebbero una precisa varietà di lingua, cioè la lingua usata sulla rete o per chattare. Si tratta di un caso di «registrazione» (Agha, 1999, 2003, 2005), ossia di fissazione di una varietà (o registro) di lingua percepita come autonoma e nuova dalla comunità di parlanti. La registrazione ha chiaramente a che vedere con la nozione di ideologia perché è un processo che scaturisce da ciò che i parlanti pensano di alcuni fatti di lingua. La definizione di registrazione è infatti la seguente:

[...] an ideological process whereby speakers' perceptions of linguistic variation, social structure, and other pertinent concepts are put to use in construing practices as group- and/or variety-specific. (Squires, 2010: 460)

Ma la definizione che meglio coglie l'emergere della registrazione come un processo, è quella di Agha il quale sostiene, in diversi articoli, che:

A register is: «a linguistic repertoire that is associated, culture internally, with particular social practices and with persons who engage in such practices». (Agha, 1999: 216; corsivi aggiunti)

Enregisterment is a process by which a new «register» emerges in the linguistic repertoire of a community: «a process whereby distinct forms of speech come to be socially recognized (or enregistered) as indexical of speaker attributes by a population of language users». (Agha, 2005: 38; corsivi aggiunti)

In Italia però la registrazione della lingua della rete (ancorché come processo non sia né chiaro né a uno stadio avanzato) avviene nella prospettiva del confronto tra lingua standard e lingua della rete e con la valutazione della seconda come varietà «decadente» che a lungo andare e praticata con insistenza e in modo esclusivo danneggerà le capacità linguistiche delle nuove generazioni. 
Il processo di registrazione nel caso della varietà della rete è originato sia dalle ideologie linguistiche sia dalle ideologie sulle tecnologie. In generale una varietà definita «lingua della rete» si sta «registrando» nell'immaginario dei vari paesi occidentali come una varietà non standard, inferiore o comunque degenerata rispetto alla lingua standard. L'idea delle degenerazione della lingua dovuta all'uso diffuso delle tecnologie trova riscontro, ad esempio, nell'ampio elenco di pagine e di gruppi di facebook (il social media più diffuso attualmente con circa 1,3 miliardi di utenti nel mondo, dati del 2013) che denigrano la netspeak. In Italia, poiché, come anticipato, non si è stabilizzato alcun termine per designare la lingua della rete, i gruppi online comunque intervengono contro i danni causati dal diffondersi delle nuove tecnologie e si fa riferimento alla difesa della lingua italiana tout court (spesso le pagine che trattano queste tematiche inseriscono nei titoli espressioni del tipo salviamo/ difendiamo/conserviamo la lingua italiana oppure aspetti specifici, esempio salviamo il congiuntivo, la virgola; a conferma del tono da crociata che questi temi suggeriscono).

Accanto alla posizione critica e denigratoria assunta dai media, anche il discorso accademico sulla lingua dei nuovi media ha spesso avallato l'idea deterministica secondo cui la comunicazione in internet è un ibrido tra scritto e parlato. In questo caso l'enfasi nello studio della lingua della rete è stata posta sul medium e sul fatto che esso alimenta il formarsi di una nuova varietà di lingua. I discorsi accademici a loro volta non solo hanno descritto la lingua usata nella CMC ma hanno contribuito alla sua registrazione. Per l'inglese la stabilizzazione di una varietà di inglese di internet deve molto ai lavori di David Crystal. Per l'italiano in verità i giornali non sembrano avere coltivato un rapporto privilegiato con nessun esponente dell'accademia su questo tema. Solo il parere dell' Accademia della Crusca è rimbalzato sulle pagine dei quotidiani in occasione di un convegno su SMS e lingua italiana (cf. atti del convegno Se telefonando... ti scrivo, a cura di Maraschio e De Martino, 2010).

La registrazione di internet come varietà di lingua nella stampa americana passa da un'idea di netspeak come varietà «criptica», gergale, argotica fatta di parole rare e specializzate usate dagli addetti ai lavori, da chi usa internet e in genere il computer (anni 1990-2000), quindi una lingua specialistica a una definizione (a partire dal 2000) in cui la netspeak si caratterizza per l'uso di pratiche tachigrafiche di vario tipo e per una punteggiatura e un'ortografia non standard. Inizia così la fase di sanzione del netspeak considerato come varietà non standard e stigmatizzato. Lo stigma si estende dai danni linguistici, comunicativi e culturali ai danni sociali (mescolando problemi della rete con altri problemi).

Oggi l'uso di una lingua della rete viene associato alla comunicazione giovanile tout court, all'inizio invece gli utenti della rete erano universitari, hacker, nerd, persone cioè con alto livello di istruzione e non necessariamente giovani. Il decennio 2000-2010 segna l'inizio della diffusione di una ideologia che vede nella lingua della rete e nella comunicazione digitale nel suo complesso l'origine di molti mali e dunque l'oggetto di una preoccupazione diffusa da parte della opinione pubblica (Squires, 2010: 468). 
Nella stampa italiana manca invece questa periodizzazione e di rete si è iniziato a parlare in un'epoca più ravvicinata quando già la rete era diventato un fenomeno di diffusione più massiccia e popolare.

\section{Italiano della rete nella stampa}

Il corpus è costituito da 30 articoli pubblicati tra il 2008 e il 2013, per un totale di circa 17.000 parole. Si tratta di articoli eterogenei (pagine di approfondimenti, pagine di cultura o di tecnologia) e in qualche caso di testi provenienti da fonti online (alcune discussioni di forum e blog).

Cominciamo dall'analisi dei titoli e consideriamo innanzitutto quali categorie di analisi a) il tono del titolo, se cioè esso sia caratterizzato da un tono emotivo allarmistico oppure neutro referenziale, e b) il registro, se cioè nel titolo venga adottato un registro informale o formale.

La maggior parte degli articoli (60\%) usa un tono allarmistico e inserisce parole appartenenti al lessico catastrofico (disincanto, pericolo, shock, rischio, rischiare, scomparsa, estinzione, crisi, morire) o che denota processi degenerativi (degradare, rovinare, distruggere, lingua morta, processo) o comunque traumatici (rivoluzionare, violare). Pochi titoli ricorrono al tono neutro-referenziale. Solo un paio di titoli rimandano a una semantica positiva (aiutare, nobilitare). Talvolta (30\% dei casi) al tono emotivo allarmistico si accompagna un registro informale. Solo due articoli sono di esponenti dell'Accademia (un articolo è firmato da Giuseppe Antonelli ed è pubblicato su il Sole24ore, un altro è firmato da Cesare Segre ed è pubblicato su www.corriere.it).

Per quanto concerne le tematiche affrontate dai vari articoli, si possono sintetizzare e raggruppare come segue:

- SMS e internet peggiorano la lingua/migliorano la lingua (il filone di articoli esce tra il 2007 e il 2010 ed è collegato a due eventi Crusca, un convegno sul tema e poi l'uscita del volume di atti del convegno);

- internet sta rovinando il mondo (determinismo tecnologico);

- la globalizzazione e internet faranno sparire la lingua italiana dal web (filone di articoli del 2012 collegato al rapporto CNR sulle tecnologie linguistiche a supporto delle lingue in internet);

- la volgarità imperante nella lingua e tra i giovani (articolo di Segre);

- apporti di internet alla creazione di neologismi (articolo sulla netspeak degli anni ‘90);

- tema del rapporto tra lingua e potere in internet (discussione su LIMES). 
Per quanto concerne la definizione della varietà di lingua usata, nel 60\% dei casi non c'è nessuna definizione, non emerge cioè il riconoscimento di una varietà autonoma di lingua. In pochi casi, minoritari, le designazioni sono generiche e valutative (linguaggio essenziale e gergale del web, linguaggio «ristretto» [nel senso di abbreviato], linguaggio settoriale, lingua tecnologica, italiano deglisms, gergo articolato, pseudo nuova lingua della rete, scrittura personale in rete). L'espressione più generica si trova nel titolo che riporto qui di seguito: Ma allora, come si può difendere l'italiano da queste nuove forme? dove la lingua usata nel web viene designata in modo semplicistico e generico come «nuove forme». In due soli articoli si usa una definizione più precisa: nel primo si parla di italiano del web o e-taliano (l'autore dell'articolo è Giuseppe Antonelli) e nel secondo si parla di social lingua (nel senso di lingua settoriale diffusa dai e sui social network e infarcita di anglicismi).

La lingua della rete, non solo non è definita in modo preciso e univoco, ma viene spesso anche rappresentata in modo caricaturale. Si veda l'incipit dell'articolo tratto dal blog associato al giornale wired.it in cui si utilizzano diverse sigle tipiche della scrittura in rete (le sigle sono glossate a fine articolo) per formare una frase:

«OMG!, IMHO saremo BFF, tu mi fai LOL».

Non sono impazzito, ho soltanto costruito una frase di senso compiuto* con i quattro vocaboli appena ammessi nel prestigioso Oxford English Dictionary. Oddio, chiamarli vocaboli non è esatto. Si tratta di quelle abbreviazioni inventate sulla Rete che vanno tanto di moda un po' per risparmiare spazio (pensate a Twitter, dove c'è un massimo di 140 caratteri disponibili per ogni messaggio), ma molto per risparmiare tempo soprattutto fra i giovanissimi. E se due anni fa l'inclusione di OK sembrò a tutti un atto dovuto, stavolta si apre la porta ad una rivoluzione linguistica. Non sto esagerando.

* Oh mio dio, a mio modesto parere saremo amici per sempre, mi fai morire dal ridere». (Luna, 2011)

In realtà i quattro vocaboli in questione sono tutti inglesi e sono segnalati perché sono stati inseriti nell'Oxford English Dictionary. In italiano solo il quarto vocabolo, $L O L$, è entrato come prestito, è conosciuto e ha ampia diffusione. Ciò che è evidente è che l'autore accompagna la notizia con espressioni e toni che vogliono evocare senso del ridicolo: «chiamarli vocaboli non è esatto». In realtà queste più che abbreviazioni nel senso di parole tronche o private di alcune lettere (come cmq per comunque, tt per tutto, sn per sono, xò per però, oppure foto per fotografia) sono delle sigle, cioè sequenze di lettere che sostituiscono ciascuna una parola, e le cui parole originarie - ridotte alla sola iniziale - a loro volta costituiscono sintagmi, frasi o comunque espressioni polirematiche (OMG: Oh My God; IMHO: In My Humble Opinion; BFF: Boy Friend Forever; LOL: Laughing Out Loud). Tutto sommato il processo per cui una sigla non è più percepita come tale ma diventa un vocabolo a tutti gli effetti non è per niente raro nella lingua inglese e non è legato esclusivamente ad internet: si pensi a vocaboli come RADAR (sigla di RAdio Detection And Ranging) e LASER (sigla di Light Amplification by Stimulated Emission of Radiation). Ma l'autore del post non si sofferma su queste differenze e sembra tutto sommato trattare le sigle come delle abbreviazioni comuni. 
Un altro aspetto che emerge dall'analisi del corpus è la quasi totale assenza di fonti per giustificare le affermazioni contenute negli articoli. Raramente sono citate fonti accademiche al di fuori dell'Accademia della Crusca (citata, come già detto, per tutto un filone di articoli che riguardano la lingua degli sms), dell'Accademia dei Lincei e del CNR (quest'ultimo citato negli articoli che commentano il rapporto sulle tecnologie linguistiche). In due casi gli articoli riferiscono le affermazioni contenute a un personaggio famoso (Elton John) o a uno specialista intervistato (l'enigmista Stefano Bartezzaghi). Ad eccezione dell'articolo di Giuseppe Antonelli, nessun articolo cita studi di linguisti.

I tratti linguistici individuati dai giornalisti italiani non sembrano far identificare una varietà di lingua specifica del web, ma piuttosto i giornalisti lamentano genericamente un decadimento della lingua italiana di cui la rete sarebbe la causa (determinismo tecnologico).

I rari esempi forniti di «imbarbarimento» della lingua sono: l'uso di abbreviazioni, acronimi, e l'emergere di un'ortografia non standard; la sintassi tendente al colloquiale (ad esempio cancellazione del congiuntivo). L'articolo di Segre evoca il tema dei registri della lingua, la scomparsa dei registri aulici e la tendenza generalizzata al turpiloquio. In qualche articolo si parla di neologismi che sono molto spesso prestiti dall'inglese (twittare, skypare).

Per quanto concerne il riferimento alla cultura giovanile si osserva che una decina di articoli (quindi poco più un terzo) mette in relazione le riflessioni - perlopiù allarmate e allarmistiche - sull'evoluzione dell'italiano con l'evoluzione (o involuzione) del mondo e della cultura giovanili, fino a menzionare esplicitamente l'ignoranza degli adolescenti. Viene in tal modo attuata quella convergenza, che già Thurlow (2005) aveva evidenziato, tra diffusione di internet, mondo dei giovani e degrado sociale, linguistico e morale.

Il linguaggio degli articoli è spesso infarcito di metafore che, non a caso, scaturiscono dal mondo della guerra (crociata contro la rete, guerra, difesa, scontro), o delle trasformazioni degenerative (degrado, crisi, deriva, allarme, imbarbarimento, perdita di purezza) o che rimandano a violenza e malattia (fare violenza alla lingua, stato di salute della lingua). Un articolo parla dei «media sul podio (sic!) degli imputati» dove - tralasciando la confusione tra «podio» e «banco degli imputati» - si fa riferimento ad un vero e proprio processo alla lingua. Discutendo del rapporto tra lingue diverse usate in internet si parla di dominio, supremazia, invasione di una lingua straniera - l'inglese - a danno dell'italiano. Ricorrono altresì metafore naturaliste e si parla di atrofizzazione delle lingue, selezione naturale, scomparsa, estinzione.

Il determinismo tecnologico anima affermazioni come le seguenti:

Ed è proprio internet, con il linguaggio tv, oltre ai giornali, a dettare le regole di questa continua evoluzione della lingua italiana. Qualcuno accusa questi modelli di incrementare un uso sciatto e improprio nella pratica scritta e parlata. (Marsella, 2010) 
Invasione di termini stranieri, impoverimento dei vocaboli, confusione nell'uso dei vari registri linguistici. Secondo 6 esperti su 10, la lingua italiana rischia oggi di perdere la sua purezza e la propria identità, faticosamente conquistata nel corso degli anni. La globalizzazione (38\%) e l'uso sempre più diffuso dei new media (26\%) sono i principali responsabili di questo processo. (Anonimo, 2012)

\section{Conclusioni}

Nonostante la nostra analisi sia stata condotta su un campione quantitativamente limitato, le linee di tendenza che emergono non sembrano del tutto inaffidabili. Le riassumerò per punti.

Innanzitutto si osserva che la stampa assume una posizione quasi sempre preoccupata e denigratoria rispetto ai nuovi media e ai nativi digitali. In genere i giornali considerano negativamente l'effetto che la diffusione dei nuovi media della comunicazione avrebbe sulla competenza linguistica soprattutto delle nuove generazioni, ritenute - forse in quanto maggiori utilizzatrici dei nuovi media maggiori responsabili del decadimento della lingua italiana.

La stampa in questo modo si erge a giudice delle tendenze linguistiche evolutive, a difensore della norma linguistica e, in mancanza di studi scientifici fondati e divulgati sulla lingua dei nuovi media, tendenzialmente influenza l'atteggiamento del mondo educativo nei confronti dei nuovi media.

I giornali italiani inoltre diffondono una serie di punti di vista in merito ai nuovi media che intrecciano la loro presunta pericolosità «sociale» (i nuovi media allontanano i giovani dalla vita reale, li isolano, li confondono attirandoli verso un mondo appunto virtuale, dove tutti gli inganni sono possibili) con la pericolosità culturale e specificatamente linguistica.

L'atteggiamento diffuso nei giornali rispetto alle nuove generazioni spesso è paternalistico. Alla critica della lingua italiana usata in rete si associano spesso critiche alle capacità di comunicare e in generale altri tratti di negatività del web collegati poi sempre al mondo giovanile. In realtà non è assodato che i problemi di comunicazione siano una prerogativa giovanile, ciononostante la stampa contribuisce a diffondere uno stereotipo negativo dei giovani. Molte accuse riferite ai giovani peccano di semplificazione: i giovani mostrano maggiore creatività degli adulti e comunque la capacità comunicativa va considerata anche in funzione dei fattori socio demografici e dei livelli di istruzione.

L'ideologia linguistica della stampa italiana rispetto alla lingua italiana della rete è tesa in linea di massima a supportare e costruire una visione negativa di questa varietà, ma si confondono diversi piani: non c'è una chiara distinzione tra i diversi problemi che scrivere in rete potrebbe comportare (problemi relativi alla conoscenza e all'uso del codice linguistico in senso stretto, da un lato, ma anche problemi di organizzazione delle conoscenze da esplicitare, dall'altro lato). Non viene identificata correttamente e tenuta separata la questione della correttezza formale da quella relativa alla scrittura e alla comunicazione tout court. 
Come si è anticipato, una varietà «lingua della rete» non esiste se non nell'immaginario collettivo supportato dall'ideologia linguistica, e alla costruzione di tale varietà la stampa contribuisce fortemente: i giornali di fatto compiono ampie generalizzazioni a vari livelli e non si preoccupano di sostanziare di una base scientifica le loro affermazioni.

In Italia i giornalisti non definiscono la lingua della rete, non la descrivono quasi per nulla, si esprimono in modo generico e superficiale in merito ad essa. Nei giornali si fa spesso riferimento all'uso sciatto della lingua e l'accusa spesso riguarda anche gli altri media, ma non ci sono particolari approfondimenti. C'è una certa confusione anche nel definire i media e nel distinguere strumenti e canali di comunicazione:

Secondo gli esperti internet (27\%), cellulari e new media (22\%) e tv (20\%) sono i media che influiscono maggiormente nella destrutturazione della lingua italiana. (Anonimo, 2012)

Meno applicabili alla situazione italiana mi sembrano altre conclusioni a cui giungeva Thurlow (2006) nel definire il rapporto della stampa americana con i nuovi media: Thurlow infatti parlava di una retorica dell'unicità e della novità (la netspeak percepita come una varietà di lingua del tutto nuova e unica); discuteva inoltre di usi statisticamente esagerati (la stampa americana accusata da Thurlow di scatenare il panico statistico gonfiando i dati di usi inadeguati della lingua); e poi di impatto negativo di internet su lingua e cultura (panico morale) fino alla feticizzazione caricaturale della lingua della rete (Thurlow, 2006).

Rispetto agli obiettivi indicati nell'introduzione dunque posso concludere che l'ideologia linguistica della stampa italiana diffonde un'idea astratta e poco scientifica sull'italiano usato in rete e non descrive, se non in toni esagerati e talvolta caricaturali, una specifica varietà di lingua.

Funziona anche per l'Italia l'effetto deterministico per cui la rete viene considerata la causa del «malcostume» linguistico - mentre, a mio modesto parere, è piuttosto il luogo in cui si manifesta un'evoluzione linguistica che trova anche altrove la sua ragion d'essere.

E infine è chiaro il contributo dei metadiscorsi della stampa al processo di registrazione di questa varietà. Inutile ribadire che una più approfondita conoscenza delle questioni, basata su articoli scientifici potrebbe cambiare la prospettiva della società sulla rete e sul tipo di comunicazione che in essa trova spazio. 


\section{Bibliografia}

Agha, Asif (1999), «Register», Journal of Linguistic Anthropology, vol. 9, n 1-2, p. 216-219.

Agha, Asif (2003), «The social life of cultural value», Language and Communication, n² 23, p. 231-273.

Agha, Asif (2005), «Voice, footing, enregisterment», Journal of Linguistic Anthropology, vol. 15, n 1 , p. 38-59.

Anonimo (2012), «Un romanzo apre il processo alla lingua italiana: per 6 esperti su 10 la lingua italiana è in crisi», Libreriamo.it, 1 ottobre 2012, disponibile su http://www.libreriamo.it/a/2754/ un-romanzo-apre-il-processo-alla-lingua-italiana-per-6-esperti-su-10-la-lingua-italiana-e-incrisi.aspx [Sito consultato il 10 maggio 2015.]

Blommaert, Jan (2006), «Language ideology», in Keith Brown (ed.), Encyclopedia of Language \& Linguistics, $2^{\text {a }}$ ed., vol. 6, Oxford, Elsevier, p. 510-522. [1ª ed., 1993.]

Durkheim, Emile (1895), Règles de la méthode sociologique, Paris, Payot.

Gal, Susan (2002), «Language Ideologies and Linguistic Diversity. Where Culture Meets Power», in László Kereszes e Sándor Maticsák (ed.), A Magyar Nyelv Idegenben, Debrecen, Debreceni Egyetem Finnugor Nyelvtudományi Tanszéke, p. 197-204.

Fiorentino, Giuliana (2013), “Wild language” goes web. New writers and old problems in the elaboration of the written code», in Emanuela Miola (ed.), Languages Go Web. Standard and non-standard languages on the Internet, Alessandria, Edizioni dell'Orso, p. 67-90.

Heath, Shirley Brice (1989), «Language ideology», in Erik Barnouw (ed.), International Encyclopedia of Communications, vol. 2, New York, Oxford University Press, p. 393-395.

Irvine, Judith T. (1989), «When talk isn’t cheap», American Ethnologist, vol. 16, n² 2, p. 248-267.

Leone, Massimo (2011), «Dall'ideologia linguistica all'ideologia semiotica. Riflessioni sulla smentita», Esercizi Filosofici, nº 6, p. 318-328.

Luna, Riccardo (2011), «Così Internet rivoluziona la lingua: e Oxford approva», Blog.Wired.it, 21 aprile 2011, disponibile su http://blog.wired.it/wewired/2011/04/21/cosi-internet-rivoluziona-la-lingua-e-oxford-approva.html. [Sito consultato il 10 maggio 2015.]

Marsella, Valentina (2010), «Da "twittare” a "fannullonismo": il lessico del Terzo Millennio, tra società e creatività», Nannimagazine.it, 21 aprile 2010, disponibile su http://www.nannimagazine.it/articolo/4569/da-twittare-a-fannullonismo-il-lessico-del-terzo-millennio-tra-societa-e-creativita [Sito consultato il 10 maggio 2015.]

Maraschio, Nicoletta e Domenico De Martino (ed.) (2010), Se telefonando... ti scrivo. L'italiano al telefono, dal parlato al digitato, Firenze, Accademia della Crusca.

Rumsey, Alan (1990), «Wording, meaning and linguistic ideology», American anthropologist, n 92, p. 346-361. 
Silverstein, Michael (1977), «Cultural prerequisites to grammatical analysis», in Muriel Saville-Troike (ed.), Linguistics and anthropology, Washington (D.C.), Georgetown University Press, p. 139-151.

Silverstein, Michael (1979), «Language structure and linguistic ideology», in Paul R. Clyne, William F. Hanks e Carol L. Hofbauer (ed.), The Elements. A parasession on linguistic units and levels, Chicago, Chicago Linguistic Society, p. 193-247.

Silverstein, Michael (1992), «The uses and utility of ideology. Some reflections», Pragmatics, n 2-3, p. 311-323.

Squires, Lauren (2010), «Enregistering internet language», Language in Society, n 39, p. 457-492.

Thurlow, Crispin (2003), «Generation Txt? The sociolinguistics of young people's text-messaging», Discourse Analysis Online, vol. 1, no 1, disponibile su www.shu.ac.uk/daol/articles/v1/n1/a3/thurlow2002003-paper.html. [Sito consultato il 3 febbraio 2015.]

Thurlow, Crispin (2005), «Deconstructing adolescent communication», in Angie Williams e Crispin Thurlow (ed.), Talking Adolescence. Perspectives on Communication in the Teenage Years, New York, Peter Lang, p. 1-20.

Thurlow, Crispin (2006), «From statistical panic to moral panic. The metadiscursive construction and popular exaggeration of new media language in the print media», Journal of Computer Mediated Communication, vol. 11, n³, p. 667-701.

Thurlow, Crispin (ed.), (2009), Young People, Mediated Discourse and Communication Technologies, numero tematico del Journal of Computer Mediated Communication, vol. 14, $n^{\circ} 4$.

Thurlow, Crispin e Susan McKay (2003), "Profiling "New" Communication Technologies in Adolescence», Journal of Language and Social Psychology, n²2, p. 94-103.

Woolard, Kathryn A. (1992), «Language ideology. Issues and approaches», Pragmatics, n² 2-3, p. 235249.

Woolard, Kathryn A. (1998), «Introduction. Language ideology as a field of inquiry», in Bambi B. Schieffelin, Kathryn A. Woolard e Paul V. Kroskrity (ed.), Language ideologies. Practice and theory, New York/Oxford, Oxford University Press, p. 3-49. 